\title{
Function and evolution of ankylosaur dermal armor
}

Shoji Hayashi, Kenneth Carpenter, Torsten M. Scheyer, Mahito Watabe, and Daisuke Suzuki Acta Palaeontologica Polonica 55 (2), 2010: 213-228 doi: http://dx.doi.org/10.4202/app.2009.0103

Ankylosaurs have spike-, plate-, and club-shaped osteoderms probably used as defensive and/or offensive weapons. Previous studies have proposed the evolution and function of small ankylosaur osteoderms, but histological variations in their defensive weapons are little known. Here, we provide comparisons of the internal structures in defensive weapons of ankylosaurs, which shed light on understanding their evolutionary history and function. Histological features of spikes, plates, and clubs are similar to those of small osteoderms in having thin compact bone, thick cancellous bone with large vascular canals, and abundant collagen fibers.Aprevious study demonstrated that each of the three groups of ankylosaurs (the Polacanthidae, Nodosauridae, and Ankylosauridae) have distinct arrangements of collagen fibers in small osteoderms. This study shows that spikes and clubs of ankylosaurs maintain the same characteristic features for each group despite the differences in shapes and sizes. These histological similarities suggest that various types of osteoderms in ankylosaurs retained the thin compact bone and abundant fiber structures of the small osteoderms during their evolution. Polacanthid spikes show thin compact bone, with less collagen fibers than in spikes of nodosaurids and spikes and clubs of ankylosaurids. Also, ankylosaurid plates with hollow bases are very thin in morphology and show thin compact bone. These results imply that the bone strengths of polacanthid spikes and ankylosaurid plates are lower than spikes and clubs of other ankylosaurs, indicating that they may be used more probably as display and/or thermoregulation rather than as weapons. It is thus probable that ankylosaur armor in general played more than just a defensive role.

Key words: Ankylosauria, Thyreophora, dermal armor, bone histology, evolution, growth, function.

Shoji Hayashi [showji@mail.sci.hokudai.ac.jp], Division of Earth and Planetary Sciences, Graduate School of Science, Hokkaido University, Sapporo, 060-0810, Japan; Kenneth Carpenter [ken.carpenter@dmns.org], Department of Earth Science, Denver 42 Museum Nature \& Science, 2001 Colorado Boulevard, Denver, CO 80205, U.S.A; Torsten M. Scheyer [tscheyer@pim.uzh.ch ], Paleontological Institute and Museum, University of Zurich, Karl Schmid-Strasse 4, CH-8006 Zurich, Switzerland; Mahito Watabe [moldavicum@pa2.so-net.ne.jp ], Center for Paleobiological Research, Hayashibara Biochemical Laboratories, Inc., Okayama 700-0907, Japan; Daisuke Suzuki [daisuke@ sapmed.ac.jp], Department of Anatomy, School of Medicine, 48 Sapporo Medical University, Sapporo 001-0810, Japan. 
This is an open-access article distributed under the terms of the Creative Commons Attribution License (for details please see creativecommons.org), which permits unrestricted use, distribution, and reproduction in any medium, provided the original author and source are credited.

For Full text $(1,720.9 \mathrm{kB})$ 